\title{
8-羟基异香豆素的合成方法研究
}

\author{
郭冬冬 ${ }^{a}$ 张武霞 ${ }^{a}$ 王永强 ${ }^{*}, b$ \\ $\left({ }^{a}\right.$ 山西农业大学文理学院 山西太谷 030801) \\ ( ${ }^{b}$ 西北大学化学与材料科学学院 西安 710069)
}

\begin{abstract}
摘要 对 8-羟基异香豆素的合成方法进行研究. 以麦氏酸为原料, 通过傅克酰基化, 羰基保护, 酯基还原, 脱保护, 得 到关键中间体 3-乙炔基-2-(羟甲基)环已-2-烯-1-酮，再经酸性环化加成，环已烯酮环的芳构化，2,3-二氯-5,6-二氧对苯醌 (DDQ)氧化等步骤, 合成得到 8-羟基异香豆素. 合成的化合物结构经过 ${ }^{1} \mathrm{H} N M R,{ }^{13} \mathrm{C} N M R, I R, H R M S$ 表征.

关键词 异香豆素; 8-羟基异香豆素; 吡喃; 芳构化; 合成方法
\end{abstract}

\section{Study on Synthetic Approach to 8-Hydroxyisocoumarin}

\author{
Guo, Dongdong ${ }^{a} \quad$ Zhang, Wuxia ${ }^{a} \quad$ Wang, Yongqiang ${ }^{*, b}$ \\ $\left({ }^{a}\right.$ College of Arts and Sciences, Shanxi Agricultural University, Taigu, Shanxi 030801) \\ ( ${ }^{b}$ Department of Chemistry \& Materials Science, Northwest University, Xi'an 710069)
}

\begin{abstract}
A new method for the synthesis of 8-hydroxyisocoumarin is reported. 8-Hydroxyisocoumarin was synthesized from Meldrum's acid by Friedel-Crafts acylation, carbonyl protection, ester reduction and deprotection. The key intermediate 3-ethynyl-2-(hydroxymethyl)cyclohex-2-en-1-one was synthesized by acidic cycloaddition, aromatization of cyclohexanone ring and 2,3-dicyano-5,6-dichlorobenzoquinone (DDQ) oxidation. The structures were characterized by ${ }^{1} \mathrm{H}$ NMR, ${ }^{13} \mathrm{C}$ NMR, IR, and HRMS.

Keywords isocoumarin; 8-hydroxyisocoumarin; pyran; aromatization; synthetic method
\end{abstract}

香豆素类化合物，特别是异香豆素，是一类重要的 天然内酯, 具有广泛的药理作用 ${ }^{[1,2]}$. 异香豆素还作为结 构单元大量存在于具有强大生物活性的天然和合成化 合物中 ${ }^{[3]}$, 这些化合物具有抗真菌、抗炎、抗菌、植物 毒性及细胞毒性等活性 ${ }^{[4]}$. 异香豆素也是合成各种重要 杂环和碳环化合物的有用中间体, 包括异喹啉、异色烯、 芳香类化合物等 ${ }^{[5]}$. 特别是 8 -羟基异香豆素, 这是一类 具有抗真菌、植物毒性和抗菌活性的天然化合物 ${ }^{[6]}$. 图 1 中为几个具有代表性的 8-羟基香豆素类化合物 ${ }^{[6]}$, 其中, 从杜仲叶中分离得到的 Thunberginol A 因其抗糖尿病特 性、抗过敏和抗菌作用而成为明星分子 ${ }^{[4-6]}$.

8-羟基异香豆素类化合物广泛的药理和生理活性, 引起合成科学家持续的兴趣. 这类化合物的合成方法已 被广泛研究, 文献中有几篇优秀的文章综述了关于异香 豆素类化合物的分离和合成方法 ${ }^{[1,2,5,7,8]}$. 异香豆素衍生<smiles>O=c1occc2cccc(O)c12</smiles>

1

8-hydroxy isocoumarin<smiles>Cc1oc(=O)c2c(O)cccc2c1C</smiles>

oospolactone<smiles>O=c1oc(-c2ccc(O)c(O)c2)cc2cccc(O)c12</smiles><smiles>COc1ccc(C2Cc3cccc(O)c3C(=O)O2)cc1O</smiles>

图 18 -羟基异香豆素类化合物

Figure 1 8-Hydroxyisocoumarin

物一般是通过邻炔基苯甲酸/酯或苯甲酰胺的环化合 成 ${ }^{[5,9]}$, 但是，一些传统或过渡金属催化的经典合成方法

\footnotetext{
* Corresponding author. E-mail: wangyq@nwu.edu.cn

Received June 3, 2019; revised July 22, 2019; published online August 7, 2019.

Project supported by the National Natural Science Foundation of China (No. 31800678), the Shanxi Excellent Doctor Grant Award (No. SXYBKY201737) and the Science and Technology Innovation Project of Shanxi Agricultural University (No. 2017YJ38).

国家自然科学基金(No. 31800678)、山西省优秀博士来晋工作奖励(No. SXYBKY201737)和山西农业大学科技创新基金(No. 2017YJ38)资助项目.
} 
反应步骤多, 反应条件苛刻. 为了克服这些限制, 实现 异香豆素的结构多样化的合成, 文献报道了钯催化的 2烯丙基苯甲酸的环化反应 ${ }^{[10 ~ 12]}$. 合成这些化合物的有 效方法也存在一些限制. 因为大多数报道的合成方法涉 及多个步骤, 使用过渡金属作为催化剂, 并且需要昂贵 的试剂或催化剂和苛刻的反应条件 ${ }^{[13 \sim 15]}$. 鉴于这些局 限性, 对于 8-差基异香豆素, 开发一种有效的合成方法 是非常需要的. Majetich 等 ${ }^{[6]}$ 以 3-乙氧基环己- 2-烯-1-酮 为起始原料, 合成了 6-羟基异香豆素和 8-羟基异香豆 素, 这是一种有效的方法. 我们设计出一种合成 8-羟基 异香豆素类化合物的新方法. 以戊二酸䣶 $(3)$ 为原料, 首 先与双三甲基硅基乙炔在无水三氯化铝作用下发生类 似的 Friedel-Crafts 反应生成取代硅炔酸 $\mathbf{4}$, 然后 4 与麦 氏酸进行缩合反应得到化合物 $\mathbf{5}$, 化合物 $\mathbf{5}$ 与乙醇反应, 脱去一份子丙酮和一分子二氧化碳环合制得 $\beta$ 酮酸酯 $\mathbf{6}$, 然后化合物 6 经乙二醇保护得到化合物 7, 7 经 DIBAl-H 还原酯基, 在酸性条件下脱去乙二醇保护基, 在碳酸钾, 甲醇溶液中脱去三甲基硅基保护基得到化合物 $\mathbf{1 0}$ 后, 10 参照文献[6]合成环已烯酤 2, 2 经过芳构化, DDQ 氧化合 成 8-羟基异香豆素 1 (Scheme 1).

\section{1 结果与讨论}

以戊二酸䣶为原料, 在无水 $\mathrm{AlCl}_{3}$ 作用下经过类 Friedel-Crafts 反应，高收率地得到硅炔酸 4(收率 95\%以 上), 主要是因为双三甲基硅炔富电子有较高反应活性. 随后化合物 $\mathbf{4}$ 与麦氏酸反应脱水缩合得到化合物 $\mathbf{5}$, 收 率 $90 \%$ 以上. 在乙醇回流条件下，化合物 5 脱去一分子 $\mathrm{CO}_{2}$ 和丙酮, 同时环合合成 $\beta$ 酮酸酯 6, 收率 $85 \%$. 化合 物 6 中羰基和酯基同时存在, 所以先保护羰基，再还原 酯基，以乙二醇作为保护基保护酮羰基. 在合成 7 的过 程中, 在乙二醇、对甲苯磺酸和甲苯回流的条件下，不 能得到目标产物; 而用乙二醇、对甲苯磺酸(PTSA)和原 甲酸三甲酯，在室温条件下以 $70 \%$ 的收率合成化合物 7, 7 经 DIBAl-H 还原得到醇 8 的同时还有副产物 9, 这使 得化合物 8 的合成收率 $(70 \%)$ 下降. 所以用比乙二醇亲 核性强的乙二硫醇作为化合物 6 的羰基保护基，以 $85 \%$ 的收率得到乙二硫醇保护的产物 11; 接着化合物 11 经 过 DIBAL-H 还原得到化合物 12, 没有发现明显的副产 物，收率 90\%. 但是在按合成路线脱保护基的时候，化 合物 12 难以脱除保护基，所以继续用化合物 8 在对甲苯

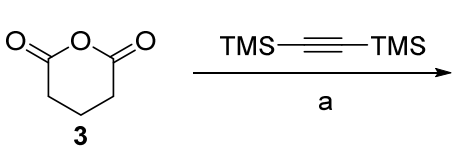

3<smiles>CCOC(=O)C1=C(C#CC(C)(C)C)CCCC1=O</smiles>

6
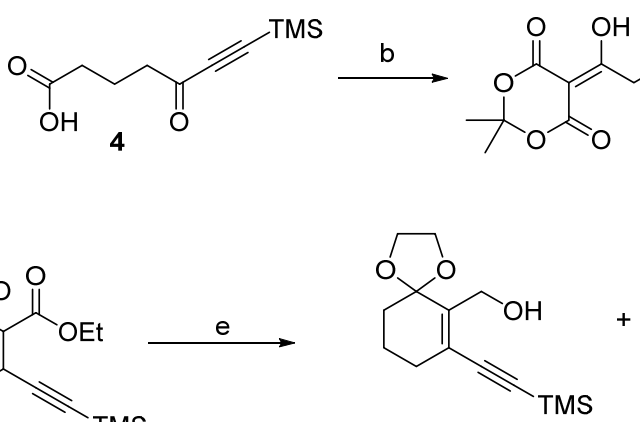

$870 \%$ major

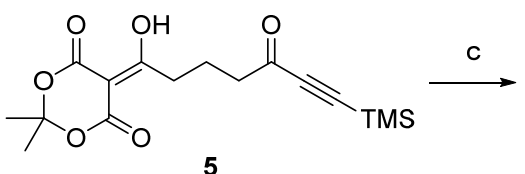

5

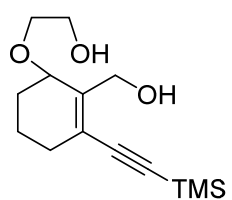

$\mathbf{8 : 9}=3.5: 1$

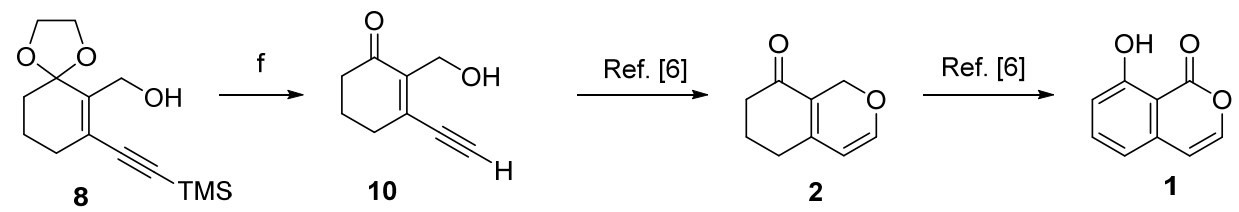<smiles>CCOC(=O)C1=C(C#CN)CCCC1=O</smiles>

6

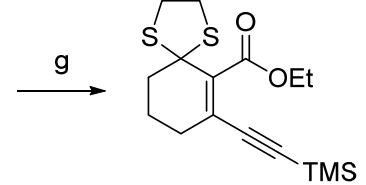

11

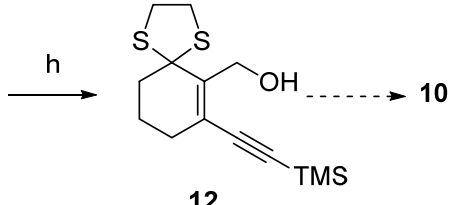

12

Reagents and conditions: (a) $\mathrm{AlCl}_{3}, \mathrm{CH}_{2} \mathrm{Cl}_{2}, 0{ }^{\circ} \mathrm{C}, 3 \mathrm{~h}$, r.t., $22 \mathrm{~h}, 95 \%$; (b) meldrum's acid, DCC, DMAP, $\mathrm{CH}_{2} \mathrm{Cl}_{2}$, r.t., 18 h, $90 \%$; (c) EtOH, reflux, $85 \%$; (d) PTSA, $\mathrm{HOCH}_{2} \mathrm{CH}_{2} \mathrm{OH}$, r.t., 18 h, $70 \%$; (e) DIBAL-H, $0{ }^{\circ} \mathrm{C}, 3 \mathrm{~h}, 8,70 \%, 9$, 20\%; (f) i) PTSA, toluene, r.t., 8 h; ii) $\mathrm{K}_{2} \mathrm{CO}_{3}$, $\mathrm{MeOH}$, r.t., 0.5 h; $60 \%$, two steps; (g) $\mathrm{HSCH}_{2} \mathrm{CH}_{2} \mathrm{SH}, \mathrm{MeOH}, \mathrm{BF}_{3} \cdot \mathrm{Et}_{2} \mathrm{O},-78{ }^{\circ} \mathrm{C}$, 40 min, r.t., 8 h, $85 \%$; (h) DIBAL-H, $\mathrm{CH}_{2} \mathrm{Cl}_{2}, 90 \%$.

图式 1 合成路线

Scheme 1 Synthetic route 
磺酸和甲苯溶液中脱乙二醇保护基，随后产物直接在甲 醇和 $\mathrm{K}_{2} \mathrm{CO}_{3}$ 中以 $60 \%$ 的收率得到关键化合物环己烯酮 10; 接着化合物环己烯酮 10 可参照文献[6]的方法合成 化合物 8-羟基异香豆素(1).

\section{2 结论}

综上所述，从简单的原料合成了 3-乙炔基-2-(羟甲 基)环己-2-烯-1-酮为关键中间体，在此基础上合成 8-差 基异香豆素, 为此类化合物的形式合成提供了一条新的 合成路线.

\section{3 实验部分}

\section{1 仪器与试剂}

${ }^{1} \mathrm{H}$ NMR 和 ${ }^{13} \mathrm{C}$ NMR 由 Bruker ascend-400 型核磁共 振仪测定, 内标为 TMS; IR 由 Bruker TENSOR 27 型红 外波谱仪测定, $\mathrm{KBr}$ 压片; 高分辨质谱(HRMS)用布鲁克 Micro TOF-Q II, 电离源(ESI)测定. 纯化用硅胶柱层析.

\section{2 实验方法}

\subsubsection{5-氧-7-(三甲基硅基)庚-6-炔酸(4)的合成}

参考文献[16], 在冰浴下, 向戊二酸酐 3 (1.14 g, 10 $\mathrm{mmol}$ )和双(三甲基硅基)乙炔(1.70 g, $10 \mathrm{mmol}$ )的 $\mathrm{CH}_{2} \mathrm{Cl}_{2}$ $(85 \mathrm{~mL})$ 溶液中, 分多次加入研细的无水 $\mathrm{AlCl}_{3}(1.40 \mathrm{~g}$, $10.5 \mathrm{mmol})$. 该混合物先在冰浴下搅拌 $3 \mathrm{~h}$, 接着在室温 搅拌 $22 \mathrm{~h}$. 在冰浴下, 向深棕褐色粘稠液体中缓慢加入 冰水, 冷却的 $1.5 \mathrm{~mol} / \mathrm{L}$ 的稀 $\mathrm{HCl}$ 淬灭反应. 调节 $\mathrm{pH}$ 为 1, 分液, 用 $1.5 \mathrm{~mol} / \mathrm{L}$ 的稀 $\mathrm{HCl}$ 、水和盐水洗涤有机相, 干燥有机相并浓缩, 快速硅胶柱色谱纯化 [ $\boldsymbol{V}$ (石油醚)： $V($ 乙酸乙酯 $)=2: 1$ ]得到 $2.01 \mathrm{~g}$ 深棕褐色油状物 4, 产率 95\%. ${ }^{1} \mathrm{H}$ NMR (400 MHz, $\left.\mathrm{CDCl}_{3}\right) \delta: 2.68(\mathrm{t}, J=7.2 \mathrm{~Hz}$, $2 \mathrm{H}), 2.43(\mathrm{t}, J=7.2 \mathrm{~Hz}, 2 \mathrm{H}), 2.05 \sim 1.95(\mathrm{~m}, 2 \mathrm{H}), 0.25(\mathrm{~s}$, $9 \mathrm{H}) ;{ }^{13} \mathrm{C} \mathrm{NMR}\left(100 \mathrm{MHz}, \mathrm{CDCl}_{3}\right) \delta: 186.45,178.66$, 101.46, 98.07, 43.70, 32.43, 18.33, -1.10; IR (KBr) $v$ : 2964, 2152, 1712, 1678, 1413, 1116, 853, $763 \mathrm{~cm}^{-1}$; HRMS (ESI) calcd for $\mathrm{C}_{10} \mathrm{H}_{16} \mathrm{NaO}_{3} \mathrm{Si}\left[\mathrm{M}+\mathrm{Na}{ }^{+}\right.$ 235.0761, found 235.0762.

3.2.25-[1-羟基-5-氧代-7-(三甲硅基)庚-6-炔-1-亚叉 基]-2,2-二甲基-1,3-二氧六环-4,6-二酮(5)的合成

参考文献 [17], 向 Meldrum's 酸 ${ }^{[18]}(785 \mathrm{mg}, 5.5$ mmol)、DMAP (190 mg, $1.56 \mathrm{mmol}$ )和 DCC (1.13 g, 5.5 $\mathrm{mmol})$ 的 $\mathrm{CH}_{2} \mathrm{Cl}_{2}(5 \mathrm{~mL})$ 溶液中加入 $4(1.43 \mathrm{mg}, 5.20$ $\mathrm{mmol})$ 的 $\mathrm{CH}_{2} \mathrm{Cl}_{2}(20 \mathrm{~mL})$ 溶液. 混合物在室温下搅拌 18 h. 加乙醚 $(50 \mathrm{~mL})$ 稀释反应物, 硅胶短柱过滤. 所得滤 液, 减压浓缩. 快速硅胶柱色谱纯化 $[V$ (石油醚)： $V$ ( 乙 酸乙酯) $=1: 1$ ]得到 $1.7 \mathrm{~g}$ 黄色油状液体 5 , 产率 $90 \%$.
化合物 5 不稳定，直接用于下一步反应. ${ }^{1} \mathrm{H}$ NMR (400 $\left.\mathrm{MHz}, \mathrm{CDCl}_{3}\right) \delta: 15.39$ (s, 1H), 3.11 2.98 (m, 2H), 2.80 $2.53(\mathrm{~s}, 2 \mathrm{H}), 2.98 \sim 2.13(\mathrm{~m}, 2 \mathrm{H}), 1.74(\mathrm{~s}, 6 \mathrm{H}), 0.24(\mathrm{~s}$, $9 \mathrm{H}) ;{ }^{13} \mathrm{C} \mathrm{NMR}\left(100 \mathrm{MHz}, \mathrm{CDCl}_{3}\right) \delta: 201.62,186.17$, $163.32,153.87,103.03,101.59$, 98.29, 91.45, 44.10, 37.41, 26.68, 21.22, - 0.91; HRMS (ESI) calcd for $\mathrm{C}_{16} \mathrm{H}_{22}$ $\mathrm{NaO}_{6} \mathrm{Si}[\mathrm{M}+\mathrm{Na}]^{+}$361.1078, found 361.1088.

3.2 .36 -氧代-2-[(三甲硅基)乙炔基]环已-1-烯-1-甲酸 乙酯(6)的合成

参考文献[19], 将化合物 5 (2.3 g, $6 \mathrm{mmol})$ 溶于无水 乙醇 $(20 \mathrm{~mL})$ 中, 加热回流 $1 \mathrm{~h}$. 减压蒸去乙醇, 浓缩, 剩 余物快速硅胶柱色谱纯化 $[V$ (石油醚) $: V($ 乙酸乙酯 $)=$ 4：1]得到 $1.35 \mathrm{~g}$ 黄色油状液体 6, 产率 $85 \%$. ${ }^{1} \mathrm{H}$ NMR $\left(400 \mathrm{MHz}, \mathrm{CDCl}_{3}\right) \delta: 4.30(\mathrm{q}, J=8.0 \mathrm{~Hz}, 2 \mathrm{H}), 2.53(\mathrm{t}, J=$ $6.0 \mathrm{~Hz}, 2 \mathrm{H}), 2.46(\mathrm{t}, J=6.8 \mathrm{~Hz}, 2 \mathrm{H}), 1.99 \sim 2.06(\mathrm{~m}, 2 \mathrm{H})$, $1.32(\mathrm{t}, J=7.2 \mathrm{~Hz}, 3 \mathrm{H}), 0.19(\mathrm{~s}, 9 \mathrm{H}) ;{ }^{13} \mathrm{C} \mathrm{NMR}(100 \mathrm{MHz}$, $\left.\mathrm{CDCl}_{3}\right) \delta: 194.34,165.33,140.43,138.30,112.21,100.62$, $61.47,37.08,30.39,21.82,14.05,-0.63$; IR (KBr) $v$ : 2960, 2900, 1735, 1672, 1589, 1304, 1232, 1051, 976, 849, $760,652 \mathrm{~cm}^{-1}$; HRMS (ESI) calcd for $\mathrm{C}_{14} \mathrm{H}_{20} \mathrm{NaO}_{3} \mathrm{Si}[\mathrm{M}+$ $\mathrm{Na}]^{+}$287.1079, found 287.1079.

3.2 .47 -[(三甲基硅基)乙炔基]-1,4-二氧螺环[4.5]癸6-烯-6-甲酸乙酯(7)的合成

参考文献[20], 将化合物 $6(5.28 \mathrm{~g}, 20 \mathrm{mmol})$ 溶于乙 二醇 $(26 \mathrm{~mL})$, 搅拌下, 加入 PTSA $(340 \mathrm{mg}, 0.20 \mathrm{mmol})$ 和原甲酸三甲酯 $(6.7 \mathrm{~mL}, 60 \mathrm{mmol})$. 在常温下反应 $18 \mathrm{~h}$, 加入冰水冷却的饱和 $\mathrm{NaHCO}_{3}$ 水溶液, 用 $\mathrm{Et}_{2} \mathrm{O}$ 萃取. 快 速硅胶柱色谱 $[V$ (石油醚)： $V$ (乙酸乙酯 $)=6: 1]$ 纯化得 到 $4.31 \mathrm{~g}$ 黄色油状物 7, 产率 70\%. ${ }^{1} \mathrm{H}$ NMR (400 MHz, $\left.\mathrm{CDCl}_{3}\right) \delta: 4.23(\mathrm{q}, J=7.2 \mathrm{~Hz}, 2 \mathrm{H}), 4.06 \sim 4.01(\mathrm{~m}, 2 \mathrm{H})$, $3.97 \sim 3.91(\mathrm{~m}, 2 \mathrm{H}), 2.25(\mathrm{t}, J=6.4 \mathrm{~Hz}, 2 \mathrm{H}), 1.86 \sim 1.78$ $(\mathrm{m}, 2 \mathrm{H}), 1.76 \sim 1.67(\mathrm{~m}, 2 \mathrm{H}), 1.32(\mathrm{t}, J=7.2 \mathrm{~Hz}, 3 \mathrm{H}), 0.14$ $(\mathrm{s}, 9 \mathrm{H}) ;{ }^{13} \mathrm{C}$ NMR $\left(100 \mathrm{MHz}, \mathrm{CDCl}_{3}\right) \delta: 166.67,137.87$, 128.14, 105.86, 102.04, 101.41, 65.44, 60.80, 33.46, 30.01, $19.59,14.04,-0.37$; IR (KBr) v: 2960, 2902, 2835, 2392, 2147, 2009, 1947, 1738, 1679, 1305, 1101, 848, $761 \mathrm{~cm}^{-1}$; HRMS (ESI) calcd for $\mathrm{C}_{16} \mathrm{H}_{24} \mathrm{NaO}_{4} \mathrm{Si}[\mathrm{M}+\mathrm{Na}]^{+}$ 331.1336 , found 331.1326 .

3.2 .5 (7-((三甲基硅基)乙炔基)-1,4-二氧螺环 [4.5]癸6-烯-6-基)甲醇(8)和 2-((2-(羟甲基)-3-((三甲硅基)乙 炔基)环已-2-烯-1-基)氧基)乙醇(9)的合成

参考文献[21], 化合物 7 (3.08 g, $10 \mathrm{mmol})$ 在 $0{ }^{\circ} \mathrm{C}$, $\mathrm{Ar}$ 保护下, 加入到无水 $\mathrm{CH}_{2} \mathrm{Cl}_{2}(100 \mathrm{~mL})$ 中, 搅拌下, 在 此温度加入 $1 \mathrm{~mol} / \mathrm{L}$ 的 DIBAL-H 的正己烷溶液 $(35 \mathrm{~mL})$. 在此温度下反应 $3 \mathrm{~h}$, 加入冰水, 稀 $1 \mathrm{~mol} / \mathrm{L}$ 盐酸溶液, 
乙酸乙酯萃取, 快速硅胶柱色谱 $[V$ (石油醚) $: V($ 乙酸乙 酯) $=4: 1$ ] 纯化得到 $1.83 \mathrm{~g}$ 无色油状物 8 (产率 70\%) 和 $0.53 \mathrm{~g}$ 无色油 9(产率 20\%).

(7-((三甲基硅基)乙炔基)-1,4-二氧螺环 [4.5]癸-6烯-6-基)甲醇(8): ${ }^{1} \mathrm{H} \mathrm{NMR}\left(400 \mathrm{MHz}, \mathrm{CDCl}_{3}\right.$ ) $\delta: 4.36$ (s, $2 \mathrm{H}), 4.15 \sim 4.08(\mathrm{~m}, 2 \mathrm{H}), 4.06 \sim 3.99(\mathrm{~m}, 2 \mathrm{H}), 2.25(\mathrm{t}, J=$ $5.3 \mathrm{~Hz}, 2 \mathrm{H}), 1.71 \sim 1.75(\mathrm{~m}, 4 \mathrm{H}), 0.20(\mathrm{~s}, 9 \mathrm{H})$; IR $(\mathrm{KBr}) v$ : 2960, 2858, 1736, 1677, 1594, $1101 \mathrm{~cm}^{-1}$; HRMS (ESI) calcd for $\mathrm{C}_{14} \mathrm{H}_{22} \mathrm{NaO}_{3} \mathrm{Si}[\mathrm{M}+\mathrm{Na}]^{+}$289.1230, found 289.1236.

2-((2-(羟甲基)-3-((三甲硅基)乙炔基)环已-2-烯-1基)氧基)乙醇(9): ${ }^{1} \mathrm{H} \mathrm{NMR}\left(400 \mathrm{MHz}, \mathrm{CDCl}_{3}\right) \delta$ : 4.62 $4.33(\mathrm{~m}, 2 \mathrm{H}), 4.25 \sim 4.01(\mathrm{~m}, 1 \mathrm{H}), 3.86 \sim 3.58(\mathrm{~m}, 4 \mathrm{H})$, $3.55 \sim 3.32(\mathrm{~m}, 1 \mathrm{H}), 2.27 \sim 1.98(\mathrm{~m}, 2 \mathrm{H}), 1.91 \sim 1.47(\mathrm{~m}$, $4 \mathrm{H}), 0.18 \sim 0.14(\mathrm{~m}, 9 \mathrm{H}) ;{ }^{13} \mathrm{C}$ NMR $\left(100 \mathrm{MHz}, \mathrm{CDCl}_{3}\right) \delta$ : 143.39, 120.67, 103.34, 99.41, 73.90, 70.28, 62.96, 61.78, $30.09,26.91,17.98,-0.12$; IR (KBr) v: 2901, 2597, $1677,1305 \mathrm{~cm}^{-1}$; HRMS (ESI) calcd for $\mathrm{C}_{14} \mathrm{H}_{24} \mathrm{NaO}_{3} \mathrm{Si}$ $[\mathrm{M}+\mathrm{Na}]^{+}$291.1387, found 291.1365.

3.2.6 3-乙炔基-2-(羟甲基)环已-2-烯-1-酮(10)的合成 将化合物 $8(1.33 \mathrm{~g}, 5 \mathrm{mmol})$ 溶于甲苯 $(25 \mathrm{~mL})$, 搅拌 下, 加入 PTSA (170 mg, $1 \mathrm{mmol})$. 在室温下反应 $8 \mathrm{~h}$. 加 入冰水, 乙酸乙酯萃取. 浓缩粗产物溶解在甲醇 $(10 \mathrm{~mL})$ 中, 加入碳酸钾 $(0.69 \mathrm{~g}, 5 \mathrm{mmol})$, 室温搅拌 $0.5 \mathrm{~h}$, 加饱 和氯化铵溶液, 浓缩, 除去甲醇, 乙酸乙酯萃取, 快速 硅胶柱色谱 $[V$ (石油醚) $: V$ (乙酸乙酯 $)=2: 1$ ]纯化得到 $0.45 \mathrm{~g}$ 无色油状物 10, 产率 60\%(两步). ${ }^{1} \mathrm{H}$ NMR (400 $\left.\mathrm{MHz}, \mathrm{CDCl}_{3}\right) \delta: 4.52$ (q, $\left.J=6.8 \mathrm{~Hz}, 2 \mathrm{H}\right), 3.79(\mathrm{~s}, 1 \mathrm{H})$, 2.83 (t, $J=6.8 \mathrm{~Hz}, 1 \mathrm{H}), 2.54(\mathrm{t}, J=5.9 \mathrm{~Hz}, 2 \mathrm{H}), 2.50 \sim$ $2.43(\mathrm{~m}, 2 \mathrm{H}), 2.06 \sim 2.00(\mathrm{~m}, 2 \mathrm{H})$; IR (KBr) v: 3130, 2959, 1716, 1428, 1364, 1260, 1223, 1095, $804 \quad \mathrm{~cm}^{-1}$; HRMS (ESI) calcd for $\mathrm{C}_{9} \mathrm{H}_{10} \mathrm{NaO}_{2}[\mathrm{M}+\mathrm{Na}]^{+}$173.0573, found 173.0580 .

\subsubsection{7-[(三甲基硅基)乙炔基]-1,4-二硫螺环[4.5]癸-} 6-烯-6-甲酸乙酯(11)的合成

参考文献[22], 化合物 $6(6 \mathrm{mmoL})$ 的无水 $\mathrm{MeOH}$ $(30 \mathrm{~mL})$ 溶液, 在 $-78{ }^{\circ} \mathrm{C} \mathrm{Ar}$ 保护下搅拌, 加入 $\mathrm{BF}_{3}$ ・ $\mathrm{Et}_{2} \mathrm{O}$ 溶液 $(69.5 \%, 1.2 \mathrm{~mL})$. 反应 $40 \mathrm{~min}$, 随后滴加乙二 硫醇 $(0.66 \mathrm{~mL})$, 自然恢复室温, 继续反应大约 $8 \mathrm{~h}$, 加入 乙醚和饱和 $\mathrm{NaHCO}_{3}$ 溶液, 乙酸乙酯萃取, 浓缩, 快速 硅胶柱色谱 $[V$ (石油醚) $: V$ (乙酸乙酯 $)=6: 1$ ] 纯化得到 $2.0 \mathrm{~g}$ 黄色油状物 11, 产率 85\%. ${ }^{1} \mathrm{H}$ NMR (400 MHz, $\left.\mathrm{CDCl}_{3}\right) \delta: 4.27(\mathrm{q}, J=7.2,2 \mathrm{H}), 3.43 \sim 3.38(\mathrm{~m}, 2 \mathrm{H})$, $3.35 \sim 3.27(\mathrm{~m}, 2 \mathrm{H}), 2.25(\mathrm{t}, J=6.2 \mathrm{~Hz}, 2 \mathrm{H}), 2.21 \sim 2.15$ (m, $2 \mathrm{H}), 1.95 \sim 1.80(\mathrm{~m}, 2 \mathrm{H}), 1.36(\mathrm{t}, J=7.2 \mathrm{~Hz}, 2 \mathrm{H}), 0.16$ (s, 9H); ${ }^{13} \mathrm{C}$ NMR $\left(100 \mathrm{MHz}, \mathrm{CDCl}_{3}\right) \delta: 167.05,141.63$, 123.29, 102.76, 100.13, 64.25, 60.89, 42.13, 40.68, 29.14, 21.83, 14.08, -0.27; IR (KBr) v: 2145, 1724, 1279, 1212, 1131, 1097, 1045, $843 \mathrm{~cm}^{-1}$; HRMS (ESI) calcd for $\mathrm{C}_{16} \mathrm{H}_{24} \mathrm{NaO}_{2} \mathrm{~S}_{2} \mathrm{Si}[\mathrm{M}+\mathrm{Na}]^{+}$363.0879, found 363.0882.

3.2 .8 (7-((三甲基硅基)乙炔基)-1,4-二硫螺环 [4.5]癸6-烯-6-基)甲醇(12)的合成

合成操作参照 [21], 无色油状物, 产率 $90 \% .{ }^{1} \mathrm{H}$ NMR (400 MHz, $\left.\mathrm{CDCl}_{3}\right) \delta: 4.47(\mathrm{~s}, 2 \mathrm{H}), 3.55 \sim 3.23(\mathrm{~m}$, $4 \mathrm{H}), 2.53 \sim 2.33(\mathrm{~m}, 1 \mathrm{H}), 2.28 \sim 2.03(\mathrm{~m}, 2 \mathrm{H}), 1.92 \sim 1.62$ (m, 2H), 0.21 (s, 9H); ${ }^{13} \mathrm{C}$ NMR (100 MHz, $\left.\mathrm{CDCl}_{3}\right) \delta$ : $145.18,123.50,103.73,100.17,68.45,62.40,42.94,40.50$, 29.77, 22.01, -0.22; IR (KBr) v: 2962, 2140, 1733, 1669, $1598,1426,1252,1002,848 \mathrm{~cm}^{-1}$; HRMS (ESI) calcd for $\mathrm{C}_{14} \mathrm{H}_{22} \mathrm{NaOS}_{2} \mathrm{Si}[\mathrm{M}+\mathrm{Na}]^{+}$321.0774, found 321.0771.

\section{2 .98 -差基- $1 H$-异色烯-1-酮(1)的合成}

参考文献[6], 将烯酮化合物 10 (300 mg, $2.0 \mathrm{mmol})$, 溶解在无水二氯甲烷中, 加入 $\mathrm{MsOH}(4 \mathrm{mmol})$. 搅拌下 回流 $15 \mathrm{~min}$. 冷却到室温, 用 $\mathrm{Et}_{2} \mathrm{O}(20 \mathrm{~mL})$ 稀释, 硅胶短 柱过滤, 浓缩. 在浓缩物(化合物 2, 约 $2.0 \mathrm{mmol}$ )中加入 四氢呋喃(THF) $(8 \mathrm{~mL}) .-78{ }^{\circ} \mathrm{C}$ 加入二异丙基胺基锂 (LDA) $(1.0 \mathrm{~mol} / \mathrm{L}, 2.6 \mathrm{~mL}, 2.6 \mathrm{mmol}$. 在此温度下搅拌 1 $\mathrm{h}$, 快速加入 $\mathrm{PhSeCl}(2.8 \mathrm{mmol})$ 的 THF $(1 \mathrm{~mL})$ 溶液. 在 $-78{ }^{\circ} \mathrm{C}$ 下继续反应 $0.5 \mathrm{~h}$, 薄层色谱(TLC)监测反应, 乙 酸乙酯萃取, 用水和饱和食盐水洗, 干燥, 浓缩至 20 $\mathrm{mL}$. 在室温下, 加入 $\mathrm{H}_{2} \mathrm{O}_{2}$ (质量分数为 $30 \%, 6 \mathrm{mmol}$ ) 摚 拌 $0.5 \mathrm{~h}$, 乙醚萃取, 用质量分数为 $10 \% \mathrm{NaHCO}_{3}$ 、水和 饱和食盐水依次洗涤, 干燥、浓缩, 在浓缩物中加入 1,4二氧六环 $(6 \mathrm{~mL})$ 和 DDQ (908 $\mathrm{mg}, 4 \mathrm{mmol})$, 室温搅拌, TLC 监测反应，乙醚萃取，用水和饱和食盐水洗涤，干 燥、浓缩, 硅胶柱色谱 $[V$ (石油醚) $: V$ (乙酸乙酯 $)=6 ： 1]$ 纯化得 $210 \mathrm{mg}$ 无色油 1, 产率 65\%. ${ }^{1} \mathrm{H}$ NMR (400 MHz, $\left.\mathrm{CDCl}_{3}\right) \delta: 11.00(\mathrm{~s}, 1 \mathrm{H}), 7.60(\mathrm{t}, J=8.0 \mathrm{~Hz}, 1 \mathrm{H}), 7.21(\mathrm{~d}$, $J=5.6 \mathrm{~Hz}, 1 \mathrm{H}), 6.99$ (d, $J=8.4 \mathrm{~Hz}, 1 \mathrm{H}), 6.89$ (d, $J=8.0$ $\mathrm{Hz}, 1 \mathrm{H}), 6.51(\mathrm{~d}, J=5.6 \mathrm{~Hz}, 1 \mathrm{H}) ;{ }^{13} \mathrm{C}$ NMR $(100 \mathrm{MHz}$, $\left.\mathrm{CDCl}_{3}\right) \delta: 166.14,161.60,143.79,137.29,136.66,115.88$, $115.75,108.27,107.27$; IR (KBr) v: 2926, 1686, 1457, 1384, 1314, 1255, 1225, 1168, 1115, 1048, 923, $821 \mathrm{~cm}^{-1}$; HRMS (ESI) calcd for $\mathrm{C}_{9} \mathrm{H}_{6} \mathrm{NaO}_{3}[\mathrm{M}+\mathrm{Na}]^{+}$185.0209, found 185.0202 .

辅助材料(Supporting Information) 化合物 1 和 $4 \sim 12$ 的 ${ }^{1} \mathrm{H}$ NMR 和 ${ }^{13} \mathrm{C}$ NMR 谱图. 这些材料可以免费从本刊 网站(http://sioc-journal.cn/)上下载. 


\section{References}

[1] (a) Barry, R. D. Chem. Rev. 1964, 64, 229

(b) Hill, R. A. Fortschritte der Chemie Organischer Naturstoffe/Progress in the Chemistry of Organic Natural Products, Springer, Vienna, 1986, pp. 1 78.

[2] Fang, Z.-X.; Liu, J.-Y.; Qiao, Y.-H. Chin. J. Org. Chem. 2018, 38, 1985 (in Chinese)

(房智兴, 刘巨艳, 乔艳红, 有机化学, 2018, 38, 1985.)

[3] Dong, G.; Li, C.; Liu, H. Molecules 2019, 24, 937.

[4] Neog, K.; Dutta, D.; Das, B.; Gogoi, P. Org. Lett. 2017, 19, 730.

[5] Pal, S.; Chatare, V.; Pal, M. Curr. Org. Chem. 2011, 15, 782.

[6] Majetich, G.; Grove, J. L. Heterocycles 2012, 84, 983.

[7] Narasimhan, N. S.; Mali, R. S. Synthesis 1983, 957.

[8] Napolitano, E. Org. Prep. Proced. Int. 1997, 29, 631

[9] Larock, R. C.; Doty, M. J.; Han, X. J. Org. Chem. 1999, 64, 8770

[10] Larock, R. C.; Yum, E. K.; Doty, M. J.; Sham, K. K. C. J. Org. Chem. 1995, 60, 3270.

[11] Larock, R. C.; Varaprath, S.; Lau, H. H.; Fellows, C. A. J. Am. Chem. Soc. 1984, 106, 5274.
[12] Korte, D. E.; Hegedus, L. S.; Wirth, R. K. J. Org. Chem. 1977, 42 1329.

[13] Cherry, K.; Parrain, J. L.; Thibonnet, J.; Duchêne, A.; Abarbri, M. J. Org. Chem. 2005, 70, 6669.

[14] Subramanian, V.; Batchu, V. R.; Barange, D.; Pal, M. J. Org. Chem. 2005, 70, 4778.

[15] Roy, S.; Roy, S.; Neuenswander, B.; Hill, D.; Larock, R. C. J. Comb. Chem. 2009, 11, 1128.

[16] Nayyar, N. K.; Hutchison, D. R.; Martinelli, M. J. J. Org. Chem. 1997, 62, 982.

[17] Organ, M. G.; Wang, J. J. Org. Chem. 2002, 67, 7847.

[18] Davidson, D.; Bernhard, S. A. J. Am. Chem. Soc. 1948, 70, 3426.

[19] Oikawa, Y.; Sugano, K.; Yonemitsu, O. J. Org. Chem. 1978, 43, 2087.

[20] Srikrishna, A.; Ramachary, D. B. Tetrahedron Lett. 2002, 43, 2765.

[21] Yamazaki, T.; Kobayashi, R.; Kitazume, T.; Kubota, T. J. Org. Chem. 2006, 71, 2499.

[22] Meyer, W. L.; Brannon, M. J.; Burgos, C. G. J. Org. Chem. 1985, 50,438 .

(Zhao, C.) 